\title{
The Influence of Spinal Cord Injury and Peripheral Nerve Injury on Muscle Elasticity in Contractures of the Soleus Muscle of Rats
}

J. Phys. Ther. Sci.

18: 1-3, 2006

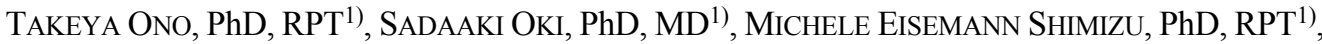 \\ AKIRA OTSUKA, PhD, RPT ${ }^{1)}$ \\ 1)Department of Physical Therapy, Faculty of Health Sciences, Prefectural University of \\ Hiroshima: 1-1 Gakuen Machi, Mihara City, Hiroshima 723-0053, Japan. \\ TEL +81848-60-1210FAX +81848-60-1226E-mail: ono@pu-hiroshima.ac.jp
}

\begin{abstract}
The purpose of this study was to investigate whether there is a difference in the influence of peripheral nerve injury and spinal cord injury on muscle elasticity in contractures of the soleus muscle of rats. Twenty-four female 8-week-old Wistar rats were divided into four groups. One group was used as a control and animals in the three experimental groups were immobilized to cause contractures. Animals in one of the experimental groups also received a spinal cord lesion and in another a peripheral nerve lesion. After four weeks, sacrificed soleus muscles were stretched to the tearing point to measure contractures. Muscle tension necessary to reach the tearing point of the lengthened soleus muscles was $5.1 \pm 0.4 \mathrm{~N}$ in the control, $3.5 \pm 0.6 \mathrm{~N}$ for the immobilized, $2.5 \pm 0.5 \mathrm{~N}$ for the spinal cord lesion, and $1.6 \pm 0.8 \mathrm{~N}$ for peripheral nerve lesion groups. These results were significantly different at the 0.05 level for all groups. Peripheral nerve injury with immobilization in a shortened position caused the severest contracture, indicating that spinal cord injury and peripheral nerve injury may have different effects on muscle contractures.
\end{abstract}

Key words: Muscle contracture, Peripheral nerve injury, Spinal cord injury

(The article was submitted Oct. 11, 2005, and was accepted Feb. 6, 2005)

\section{INTRODUCTION}

Restriction of joint range of motion (ROM) is a common clinical problem found in patients receiving therapeutic exercise. Anatomically, a muscle contracture is one of the structural factors that causes ROM restriction ${ }^{1)}$ when the muscles are immobilized in a shortened position.

Many patients who have suffered an injury to the spinal cord or peripheral nerve also develop complications in ROM restrictions. Typically, spinal cord injury causes spasticity and peripheral nerve injury causes flaccidity of the muscles.
Therefore, these muscle conditions may result in muscle contractures. However, it has not been clarified as to whether spinal cord injury and/or peripheral nerve injury influences the development of muscle contractures.

The purpose of this study was to investigate the influence of spinal cord injury and peripheral nerve injury on muscle elasticity in contractures of the soleus muscle of rats.

\section{MATERIALS AND METHODS}

Twenty-four female 8-week-old Wistar rats with 
an initial body weight of $180 \mathrm{~g}$ to $250 \mathrm{~g}$ were used. The experiments were conducted in accordance with the Prefectural University of Hiroshima Guidelines for Animal Experimentation and the U.S. National Institute of Health guidelines. The animals were housed in a temperature-controlled room at $20^{\circ} \mathrm{C}$ with a 12 hour light-dark cycle. The rats were provided free access to standard rat food and water.

Six rats were used as a control (G1). Eighteen rats out of 24 received immobilization of the soleus muscle. Under Nembutal anesthesia $(1.0 \mathrm{~mL} / \mathrm{kg})$, the soleus muscle was immobilized by unilateral pinning of the ankle joint. A wire was introduced into the tibia through the ankle joint in full plantar flexion at the calcaneus. In this position, the soleus muscle was maintained in a shortened position. These eighteen rats were divided into three groups. The first group was only immobilized at the ankle joint (G2). The second group was immobilized at the ankle joint, and spinal transections were made at the T8 level (G3). In the third group, the ankle joint was immobilized and a sciatic neurectomy was performed (G4). The duration of the immobilization was 4 weeks for all groups.

To measure the degree of muscle contracture, direct traction of the muscle was done. After sacrifice with Nembutal $(1.0 \mathrm{~mL} / \mathrm{kg})$, direct traction of the soleus muscle was done using an Autograph (AG-50KNG; Shimadzu Co, Japan). After the fixation of the leg in the Autograph, the tibia, fibula, and all lower leg muscles, except for the soleus, were cut, and the soleus was stretched to the tearing point $(10 \mathrm{~mm} / \mathrm{min})$. Individual soleus muscle traction results were expressed in length-tension curves.

During the four weeks of the experiment, the kicking movements of the opposite lower leg of the rats in G3 were tested to verify the presence of spasticity ${ }^{2)}$. At the end of the four weeks, the tension was measured at the $10-\mathrm{mm}$ lengthened position and at the tearing point of the soleus muscle, respectively. The $10-\mathrm{mm}$ lengthened position represents the normal movement from plantar flexion to dorsiflexion of the soleus muscle in a normal living rat $^{3}$.

A one-way analysis of variance was used for the analysis of the tension torque at the $10-\mathrm{mm}$ lengthened point of the soleus muscle and at the tearing point of the lengthened soleus muscle. Fisher's PLSD post hoc test was used to identify significant comparisons. The accepted alpha level of all of the statistical processing was at the 0.05 level.

\section{RESULTS}

\section{Length-tension curves of the soleus muscles}

The curves for G2 and G3 rose in the same fashion, but the G3 muscles split at a much lower tension. The G4 soleus muscles had already severed long before reaching the 10-mm lengthened position. Statistically, tension at the $10-\mathrm{mm}$ lengthened position of the soleus muscles was significantly different between G1 and G2, and G1 and G3. The tension necessary to reach the tearing point of the lengthened soleus muscles was $5.1 \pm 0.4$ $\mathrm{N}$ for $\mathrm{G} 1,3.5 \pm 0.6 \mathrm{~N}$ for $\mathrm{G} 2,2.5 \pm 0.5 \mathrm{~N}$ for $\mathrm{G} 3$, and $1.6 \pm 0.8 \mathrm{~N}$ for $\mathrm{G} 4$, showing statistical signficance for all four groups (Fig. 1).

\section{Kicking movements}

During the first week, kicking movements were

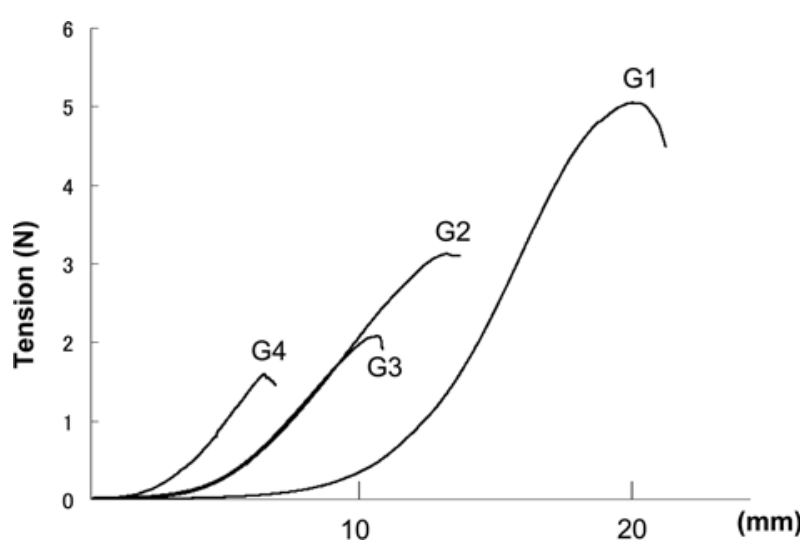

Fig. 1. Effects of spinal cord injury and peripheral nerve injury on the length-tension curve in rat soleus muscles immobilized in the shortened position. The beginning points of the curves indicate the physiological length of the soleus muscles in the completely shortened position, in vivo. The curves rise as the muscles are stretched. The tops of the curves show the tearing points due to stretch. Each curve is the average of the muscles examined in each group, G1: Control muscles, G2: Immobilized shortened soleus muscles, G3: Spinal cord injury with immobilized shortened soleus muscles, G4: Peripheral nerve injury with immobilized shortened soleus muscles. 
not observed in G3. After one week, kicking movements were clearly observed up until the end of the four weeks showing that a spinal cord injury had actually occurred. This phenomenon was the same as that reported by van de Meent et al. ${ }^{2)}$.

\section{DISCUSSION}

The causes of muscle contracture include immobilization in a shortened position ${ }^{4}$, change of muscle properties due to muscle spasticity ${ }^{5}$, and a "regular loss of contractile material" in the muscle as a result of a peripheral nerve injury6). However, a study comparing the influence of a spinal cord injury and a peripheral nerve injury on muscle elasticity in contractures of the soleus muscle of rats has not been noted in the literature. In our study, a peripheral nerve injury with immobilization in a shortened position caused the severest muscle contracture, followed by spinal cord injury, and, lastly, immobilization. We conclude that spinal cord injury and peripheral nerve injury have very different effects on the development and properties of muscle contractures.

Clinically, these results may provide very useful information for risk management during the treatment of muscle contractures. Particularly, it seems essential that clinicians should be heedful of muscle tearing when stretching the muscles of a patient with a contracture resulting from a peripheral nerve injury. Further investigation is necessary to determine if therapeutic exercise has an effect on muscle contractures caused by spinal cord injury or peripheral nerve injury.

\section{REFERENCES}

1) Trudel G, Uhthoff HK: Contractures secondary to immobility: is the restriction articular or muscular? An experimental longitudinal study in the rat knee. Arch Phys Med Rehabil, 2000, 81: 6-13.

2) van de Meent H, Hamers FP, Lankhorst AJ, et al.: New assessment techniques for evaluation of posttraumatic spinal cord function in the rat. J Neurotrauma, 1996, 13: 741-754.

3) Oki S, Shibata T, Matsuda Y, et al.: The appearance and the progression of muscle contracture in the immobilized muscle. J Physical Medicine, 1998, 9: 38-41 (in Japanese).

4) Tabary JC, Tabary C, Tardieu C, et al.: Physiological and structural changes in the cat's soleus muscle due to immobilization at different lengths by plaster casts. J Physiol, 1972, 224: 231-244.

5) O'Dwyer N, Ada L, Neilson D: Spasticity and muscle contracture following stroke. Brain, 1996, 119: 17371749.

6) Pellegrino C, Franzini C: An electron microscope study of denervation atrophy in red and white skeletal muscle fibers. J Cell Biol, 1963, 17: 327-349. 